\begin{tabular}{|c|l|}
\hline Title & Photo-induced fluorescence emission enhancement of azobenzene thin films \\
\hline Author(s) & Haruta, Osamu; Matsuo, Y asutaka; ljiro, Kuniharu \\
\hline Citation & $\begin{array}{l}\text { Colloids and surfaces. A, Physicochemical and engineering aspects, 313 314, 595-599 } \\
\text { https://doi.org/10.1016j.colsurfa.2007.05.050 }\end{array}$ \\
\hline Issue Date & 2008-02-01 \\
\hline Doc URL & http://hdl.handle.net/2115/33775 \\
\hline Type & article (author version) \\
\hline File Information & Ijiro.pdf \\
\hline
\end{tabular}

Instructions for use 


\title{
Photo-induced Fluorescence Emission Enhancement of Azobenzene Thin Films
}

\author{
Osamu Haruta ${ }^{a}$, Yasutaka Matsuo ${ }^{b}$, and Kuniharu Ijiro b, c, * \\ ${ }^{a}$ Graduate School of Science, Hokkaido University, N21W10 Kita-ku, Sapporo, \\ Hokkaido 001-0021, Japan. \\ ${ }^{b}$ Research Institute for Electronic Science (RIES), Hokkaido University, N12W6 Kita-ku, \\ Sapporo, Hokkaido 060-0812, Japan. \\ ${ }^{c}$ CREST, Japan Science and Technology Agency, 4-1-8 Honcho Kawaguchi, Saitama, \\ Japan.
}

PACS: 33.50.Dq; 61.80.Ba; 68.18.--g

Keywords: Azobenzene; Fluorescence; Monolayer; UV

* Corresponding author. Tel. +81-11-706-9360; fax: +81-11-706-9361.

E-mail address: ijiro@poly.es.hokudai.ac.jp 


\begin{abstract}
UV irradiation-induced fluorescence enhancement of thin films composed of azobenzene amphiphiles, $\mathrm{C}_{12} \mathrm{AzoC}_{10} \mathrm{~N}^{+}\left(\mathrm{AzoN}^{+}\right)$, was investigated. UV irradiation to Langmuir-Blodgett (LB) monolayers of $\mathrm{AzoN}^{+}$indicated that the fluorescence emission from an irradiated area was enhanced and the emission was independent of the aggregation structures of an azobenzene moiety. Moreover, UV irradiation to cast films of $\mathrm{AzoN}^{+}$revealed that the enhancement was increased by increasing the irradiation time. The fluorescence by excitation at $365 \mathrm{~nm}$ occurred over a wide range of wavelengths from around $400 \mathrm{~nm}$ to $650 \mathrm{~nm}$, and the emission was not attributed to the $\mathrm{J}$ aggregates of $\mathrm{AzoN}^{+}$. The mechanism for this phenomenon has not been elucidated. It may be caused by the photo-chemical reaction of the azobenzene moiety or photo-induced specific aggregates. However, UV irradiation-induced fluorescence enhancement can be applicable to optical memory devices.
\end{abstract}




\section{Introduction}

There have been many studies on the fabrication of optical memory devices that can store highly dense information within a limited area [1]. It is well known that azobenzene is a model compound for photo-switched memory devices by using their characteristic property of reversible trans-to-cis photoisomerization [2], as well as for representative dye molecules in industries [3].

In these areas, the characteristic features of fluorescence emission also play an important role in functional materials like light-emitting semiconductors [4]. With regard to the emission properties of azobenzene derivatives, a large number of studies have been reported [5], suggesting that an azobenzene unit in itself hardly fluoresces. However, it has been reported that J-aggregated azobenzene in the bilayer membrane led to two efficient fluorescence emissions at around $400 \mathrm{~nm}$ and $600 \mathrm{~nm}$ [6]. Hence, the aggregation of azobenzene unit assumed a key role for the intense fluorescence. Recently, it have been also reported that the self-assembled micelle-like aggregates of cis-azobenzene derivatives by UV illumination in solution showed aggregate size- and structure-dependent fluorescence emissions [7].

Herein we report UV irradiation-induced fluorescence enhancement in azobenzene thin films. Langmuir-Blodgett (LB) monolayers and cast films of azobenzene amphiphiles $\left(\mathrm{AzoN}^{+}\right)$were prepared and irradiated by UV light. The UV irradiation-induced fluorescence emission properties of the azobenzene thin films will be discussed.

\section{Experiment}

2. 1. Surface Pressure-Area ( $\pi$-A) isotherm measurement. 
The preparation of the azobenzene amphiphile, $\mathrm{C}_{12} \mathrm{AzoC}_{10} \mathrm{~N}^{+}\left(\mathrm{AzoN}^{+}\right)$, employed in this experiment has been reported previously [8]. An adequate amount of a $1 \mathrm{mM}$ chloroform solution of $\mathrm{AzoN}^{+}$was spread onto water purified to $18.2 \mathrm{M} \Omega \cdot \mathrm{cm}$ by a Milli-Q SP reagent system (Millipore Co.). The surface pressure-area ( $\pi$-A) isotherm was measured by using a computer controlled film balance system (FSD-300, USI). The trough size was $190 \times 90 \mathrm{~mm}^{2}$, and the temperature of the subphase was maintained at $20.0 \pm 0.1{ }^{\circ} \mathrm{C}$ during the measurement.

\section{2. Preparation of Langmuir-Blodgett (LB) monolayers on substrates.}

The $\mathrm{AzoN}^{+}$monolayer was prepared according to subsection 2.1. When the surface pressure reached $0 \mathrm{mN} / \mathrm{m}$ (without compression of the monolayer), $5 \mathrm{mN} / \mathrm{m}, 20 \mathrm{mN} / \mathrm{m}$, and $25 \mathrm{mN} / \mathrm{m}$, a clean glass substrate-immersed under the subphase before spreading the monolayer was lifted up at a speed of $5 \mathrm{~mm} / \mathrm{min}$. The transfer of the monolayers onto the substrates was performed in the vertical mode by using a LB lift controller (FSD-21, USI).

\section{3. UV-Vis absorption spectroscopy measurements of the transferred AzoN $N^{+}$} monolayers.

A quartz slide (thickness: $0.2 \mathrm{~mm}$, area: $65 \times 20 \mathrm{~mm}^{2}$ ) tapered at both sides by $60^{\circ}$ was used as a substrate for the preparation of the LB monolayers at a surface pressure of 5 $\mathrm{mN} / \mathrm{m}$ and $20 \mathrm{mN} / \mathrm{m}$. UV-Vis absorption spectra of the AzoN ${ }^{+}$monolayers deposited on the quartz substrates were measured with an optical fiber-equipped surface and interface spectrometer (SIS-50, System Instruments Co., Ltd.). The light from a $150 \mathrm{~W} \mathrm{D}_{2}$ lamp (Hamamatsu photonics) was incident on one side of the quartz substrate. Then, the light, 
which was totally reflected at the slide interface, was analyzed by a UV-Vis absorption spectrophotometer system (LB-100, JASCO) in a wavelength region of $200 \mathrm{~nm}$ to 600 nm.

2. 4. Fluorescence microscopy observation and UV irradiation to the AzoN $N^{+}$ monolayers.

Fluorescence images of the $\mathrm{AzoN}^{+}$monolayers transferred onto the glass substrates at surface pressures of $5 \mathrm{mN} / \mathrm{m}$ and $25 \mathrm{mN} / \mathrm{m}$ were acquired by a fluorescence microscope (ECLIPSE E600, Nikon) equipped with fluorescence filter sets, 40× and 100× (N.A. 1.3) oil immersion objective lenses, and a digital charge coupled device (CCD) camera (ORCA-II-ER, Hamamatsu photonics). By adjusting the aperture, an adequate area of the $\mathrm{AzoN}^{+}$monolayer was initially exposed to UV irradiation $(330-380 \mathrm{~nm})$ for $5 \mathrm{~min}$. Then, the same position of the monolayer was observed by UV-excitation (330-380 nm), B-excitation (450-490 nm), and G-excitation (510-560 nm) with approximately a three times-enlarged aperture.

Photo patterning of the $\mathrm{AzoN}^{+}$monolayer (transferred at the surface pressure of 5 $\mathrm{mN} / \mathrm{m}$ ) masked by a square mesh grid (pitch: $125 \mu \mathrm{m}$ ) was carried out by UV irradiation (240-400 nm) from a $200 \mathrm{~W} \mathrm{Hg-Xe} \mathrm{lamp} \mathrm{(Hamamatsu} \mathrm{photonics)} \mathrm{for} 5 \mathrm{~min}$.

2. 5. Fluorescence and absorption spectroscopic measurements of AzoN $N^{+}$cast films.

$100 \mu \mathrm{l}$ of a $1 \mathrm{mM}$ chloroform solution of $\mathrm{AzoN}^{+}$was cast onto a quartz substrate and dried to evaporate the chloroform. Fluorescence spectra of the cast films were measured by using a fluorescence spectroscopy system consisting of a spectrometer (CHROMEX), 
a fluorescence microscope (BX51, OLYMPUS), and a CCD camera (ANDOR DV420-OE).

UV-Vis transmission absorption spectra of the cast films were obtained by using a UV-Vis-NIR spectrophotometer (LAMBDA 900, PerkinElmer Co., Ltd.).

\section{Results and discussion}

The result of $\pi$-A isotherm measurement of $\mathrm{AzoN}^{+}$on pure water is shown in Fig.1. (b). The molecular area extrapolated to zero pressure in the condensed phase (solid phase) was calculated to be about $0.41 \mathrm{~nm}^{2} /$ molecule, which was consistent with the value reported in a previous article [9].

In the inset of Fig.1. (b), UV-Vis absorption spectra of $\mathrm{AzoN}^{+}$monolayers transferred onto the tapered quartz substrates at surface pressures of $5 \mathrm{mN} / \mathrm{m}\left(\mathrm{A} \approx 0.6 \mathrm{~nm}^{2}\right)$ and 20 $\mathrm{mN} / \mathrm{m}\left(\mathrm{A}<0.4 \mathrm{~nm}^{2}\right)$ are shown. Two strong absorption bands due to $\pi-\pi^{*}$ electronic transitions were observed at around $240 \mathrm{~nm}$ and $350 \mathrm{~nm}$. The band located at around $240 \mathrm{~nm}$ is attributed to a transition dipole moment along the short axis of the azobenzene group. The long axis transition at a lower photoexcitation energy of around $350 \mathrm{~nm}(\lambda \max )$ is strongly affected by the tilt angle of the azobenzene amphiphiles in the assemblies [10]. According to some previous reports [10,11], $\lambda \max$ of azobenzene is shifted from about $350 \mathrm{~nm}$ (monomer state) to around $300 \mathrm{~nm}$ when the parallel orientation of azobenzene, the H-aggregate, is formed. On the other hand, the head-to-tail orientation of azobenzene, the J-aggregate, gives a $\lambda \max$ of around $400 \mathrm{~nm}$. This hypsochromism and bathochromism is considered to be a result of Davydov splitting, and the details were explained well by Kasha [12]. Considering both $\pi$-A isotherm and UV-Vis absorption spectra in Fig. 1, the $\lambda \max$ of $\mathrm{AzoN}^{+}$was around 347 
$\mathrm{nm}$ in the initial liquid phase $\left(\mathrm{A} \approx 0.6 \mathrm{~nm}^{2}\right)$, indicating that the azobenzene units remained in the monomeric state. In the solid phase $\left(\mathrm{A}<0.4 \mathrm{~nm}^{2}\right)$, however, $\lambda \max$ was blue-shifted to around $332 \mathrm{~nm}$, indicating the formation of H-aggregates.

The fluorescence images of the transferred $\mathrm{AzoN}^{+}$monolayers prepared at different surface pressures of $5 \mathrm{mNm}$ and $25 \mathrm{mN} / \mathrm{m}$ were observed by UV-excitation after $5 \mathrm{~min}$ UV irradiation under the fluorescence microscope (Fig. 2. (2)). It was estimated that the intensity at a focal spot was increased up to $\approx 10000 \mathrm{~mW} / \mathrm{cm}^{2}$ through the objective lens. As shown in Fig. 2. (2), only UV irradiated areas were emitting under UV-excitation. The fluorescence emission was hardly observed outside of the UV irradiated area. In addition, the fluorescence intensities of the $\mathrm{AzoN}^{+}$monolayers prepared at each surface pressure were almost the same. However, any emissions were not observed when B- or G-irradiation was used instead of UV irradiation. As a control experiment, no fluorescence was observed at the glass substrate without the $\mathrm{AzoN}^{+}$monolayer (data not shown). Moreover, the fluorescence emissions under B- and G-excitation were also observed after the process of UV irradiation (Fig. 2. (3)). These results indicated that the UV irradiation-induced fluorescence enhancement of the $\mathrm{AzoN}^{+}$monolayers was independent of the aggregate structures reported in [6,7].

In addition to the simple patterning of the UV irradiation under the fluorescence microscope, we demonstrated a lattice-shaped patterning by UV $(240-400 \mathrm{~nm}, \approx 600$ $\mathrm{mW} / \mathrm{cm}^{2}$ ) irradiation to the $\mathrm{AzoN} \mathrm{N}^{+}$monolayer covered with a square mesh grid (pitch: $125 \mu \mathrm{m})$ as a photomask. Fig. 3 clearly shows that the lattice-shaped patterns of emissions under B- and G-excitation, which had the same pitch as the square mesh grid, were formed. On the other hand, such fluorescence and patterns were not observed when the weaker UV lamp $\left(\approx 10 \mathrm{~mW} / \mathrm{cm}^{2}\right)$ was used. And no fluorescence emission of 
the $\mathrm{AzoN}^{+}$monolayer constiting of cis azobenzene was observed (data not shown). Hence, this suggests that the observed emissions in Fig. 3, as well as Fig. 2, were not simply attributed to the cis-formed azobenzene.

Although we tried to measure the fluorescence spectra of the $\mathrm{AzoN}^{+}$monolayers to investigate the mechanism of UV irradiation-induced fluorescence enhancement, fluorescence emissions were too weak to analyze with our system. Then, the cast film of AzoN ${ }^{+}$prepared on a quartz substrate was applied to measure the fluorescence spectra by a spectrometer equipped with a microscope. By excitation at $365 \mathrm{~nm}$, a weak orange-colored fluorescence, which was centered at $600 \mathrm{~nm}$ in the fluorescence spectrum, was observed in a fluorescence image without the UV treatment (Fig. 4. (a)). As described before, J-aggregated azobenzene molecules had the emission at $600 \mathrm{~nm}$ by UV-excitation [6]. Therefore, the orange-colored fluorescence observed from the azobenzene cast films could indicate that the J-aggregated azobenzene molecules were present in the films. By UV irradiation for 150 minutes, the orange-colored fluorescence was changed into a strong blue-white fluorescence, which was centered at 500-550 nm with a wide emission wavelength (Fig. 4. (a). top).

The transmission absorption spectra of the cast films before and after UV irradiation are shown in Fig. 4. (b). The UV-Vis absorption spectrum before UV irradiation was broad with a shoulder at around $370 \mathrm{~nm}$ corresponding to the J-aggregated azobenzene. By UV irradiation, the absorption of the $\mathrm{AzoN}^{+}$cast film was significantly decreased, and any changes like the formation of the $\mathrm{J}$ aggregate in the spectra were not observed. Although the relationship between the observed emission and the conformation of azobenzene was not elucidated, the UV irradiation-induced fluorescence emissions could have possibly originated from the deformation of the azobenzene unit, as well as 
the formation of a specific aggregation. The mechanisms of this phenomenon are now under investigation by changing other conditions, e.g. substituent, temperature, intensity of UV light, and wavelength.

\section{Conclusion}

Monolayers of $\mathrm{AzoN}^{+}$transferred onto glass substrates by the LB technique at surface pressures of $5 \mathrm{mN} / \mathrm{m}, 20 \mathrm{mN} / \mathrm{m}$, and $25 \mathrm{mN} / \mathrm{m}$, were irradiated by UV light (330-380 $\mathrm{nm},>600 \mathrm{~mW} / \mathrm{cm}^{2}$ ), resulting in the enhancement of the fluorescence emissions with a wide range of excitation wavelengths. The UV irradiation-induced fluorescence enhancement was independent of the aggregation structures of the $\mathrm{AzoN}^{+}$monolayers. Moreover, UV light irradiation to the monolayers covered with a square mesh grid enabled the fabrication of a lattice-shaped pattern of fluorescence emission. Experiments using UV light irradiation to the $\mathrm{AzoN}^{+}$cast films revealed that the enhancement of fluorescence emission was increased by increasing the UV irradiation time, and the fluorescence emissions were in a wide range of wavelength from around $400 \mathrm{~nm}$ to $650 \mathrm{~nm}$. In addition, the emission was not merely attributed to the J aggregates of $\mathrm{AzoN}^{+}$. We suggest that UV irradiation-induced fluorescence enhancement of the azobenzene thin films may provide a potential route for the fabrication of new optical memory devices. 


\section{Acknowledgments}

This work was partly supported by a Grant-in-Aid for Scientific Research from the Ministry of Education, Culture, Sports, Science and Technology, Japan. 


\section{References}

[1] H. Mustroph, M. Stollenwerk, V. Bressau, Angew. Chem., Int. Ed. 45 (2006) 2016.

[2] K. Ichimura, Chem. Rev. 100 (2000) 1847.

[3] H. Zollinger, Colour Chemistry, Synthesis, Properties and Applications of Organic Dyes, VCH, Weinheim, 1987.

[4] D. C. Look, Mater. Sci. Eng., B 80 (2001) 383.

[5] H. Rau, Angew. Chem., Int. Ed. 12 (1973) 224.

[6] M. Shimomura, T. Kunitake, J. Am. Chem. Soc. 109 (1987) 5175.

[7] M. Han, M. Hara, J. Am. Chem. Soc. 127 (2005) 10951.

[8] M. Shimomura, R. Ando, T. Kunitake, Ber. Bunsenges. Phys. Chem. 87 (1983) 1134.

[9] N. Kimizuka, T. Kunitake, Colloids Surf. 38 (1989) 79.

[10] M. Shimomura, Prog. Polym. Sci. 18 (1993) 295.

[11] O. Haruta, J. Nishida, K. Ijiro, Colloids Surf., A 284-285 (2006) 326.

[12] M. Kasha, Radiat. Res. 20 (1963) 55. 


\section{Figure Captions}

Fig. 1. (a) Chemical structure of $\mathrm{C}_{12} \mathrm{AzoC}_{10} \mathrm{~N}^{+}\left(\mathrm{AzoN}^{+}\right)$, and (b) surface pressure-area $(\pi-\mathrm{A})$ isotherm of $A z o \mathrm{~N}^{+}$on water subphase. The inset shows UV-Vis absorption spectra of $\mathrm{AzoN}^{+}$monolayers deposited onto quartz substrates at surface pressure of $5 \mathrm{mN} / \mathrm{m}$ $\left(\mathrm{A} \approx 0.6 \mathrm{~nm}^{2}\right)$ and $20 \mathrm{mN} / \mathrm{m}\left(\mathrm{A}<0.4 \mathrm{~nm}^{2}\right)$.

Fig. 2. (1) Schematic illustration of the UV treatment. (2) Fluorescence images of $\mathrm{AzoN}^{+}$monolayers prepared at surface pressures of (a) $5 \mathrm{mN} / \mathrm{m}$ and (b) $25 \mathrm{mN} / \mathrm{m}$ by UV-excitation at 330-380 nm. (3) Fluorescence images of $\mathrm{AzoN}^{+}$monolayers prepared at a surface pressure of $5 \mathrm{mN} / \mathrm{m}$ by (a) B-excitation at 450-490 $\mathrm{nm}$ and (b) G-excitation at 510-560 $\mathrm{nm}$. Observation wavelength was larger than $400 \mathrm{~nm}$ for UV-excitation, 520 $\mathrm{nm}$ for B-excitation, and $590 \mathrm{~nm}$ for G-excitation.

Fig. 3. Fluorescence images of $\mathrm{AzoN}^{+}$monolayers observed by (a) B-excitation and (b) G-excitation.

Fig. 4. (a) Fluorescence spectra of $\mathrm{AzoN}^{+}$cast film. These spectra were obtained by excitation at $365 \mathrm{~nm}$ after UV treatment for $0 \mathrm{~min}$ (bottom), $60 \mathrm{~min}$ (middle), and 150 min (top). (b) Transmission absorption spectra of the $\mathrm{AzoN}^{+}$cast film before and after UV irradiation. 
(a)<smiles></smiles>

(b)

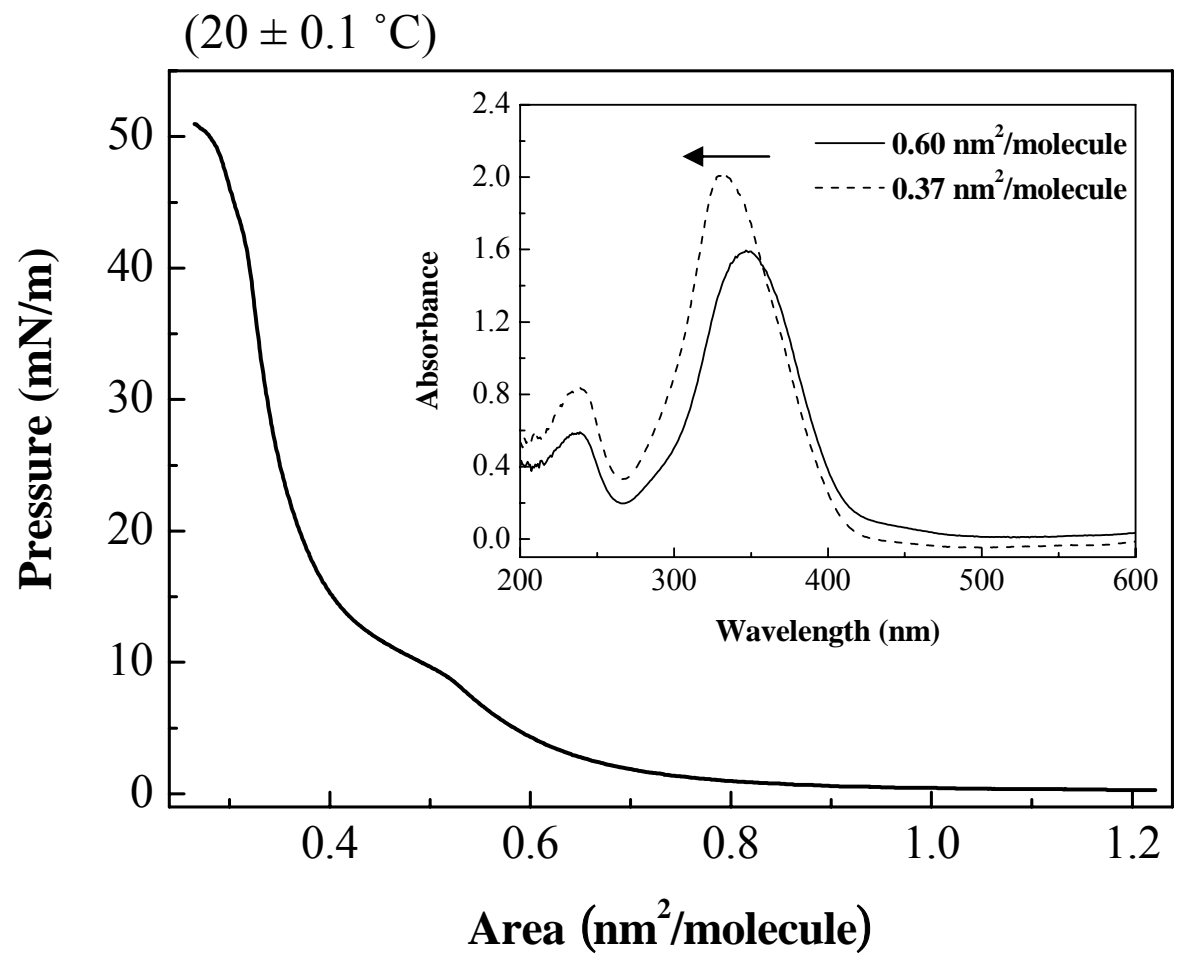

Fig. 1. O. Haruta, et al. 
(1)
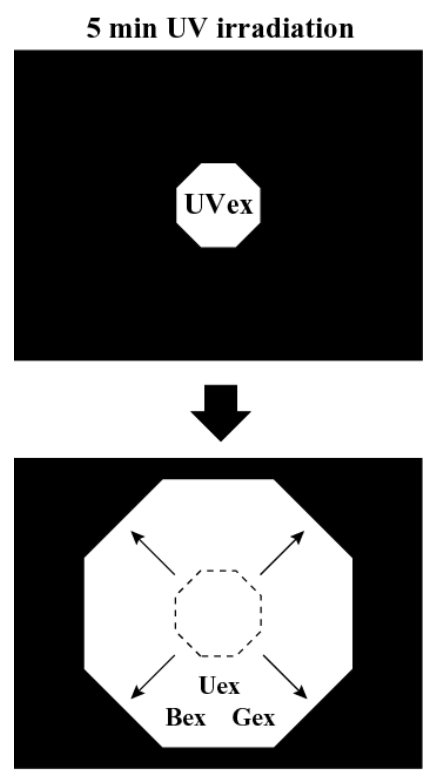

Fluorescence Observation

with enlarged aperture

(2)

$\underline{\text { U-excitation }}$

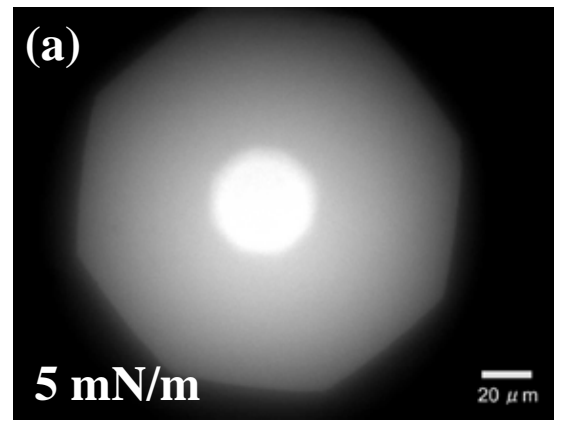

(a)

(3)

B-excitation

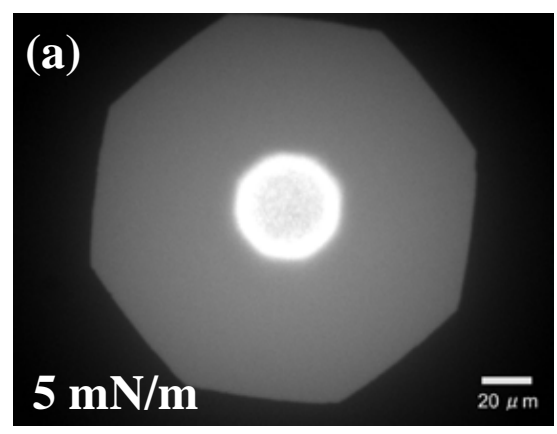

$\underline{\text { U-excitation }}$

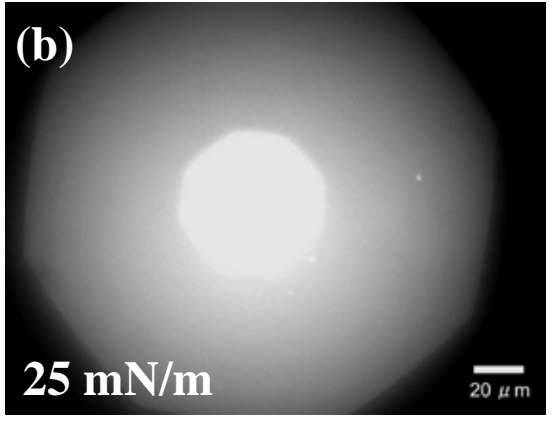

G-excitation

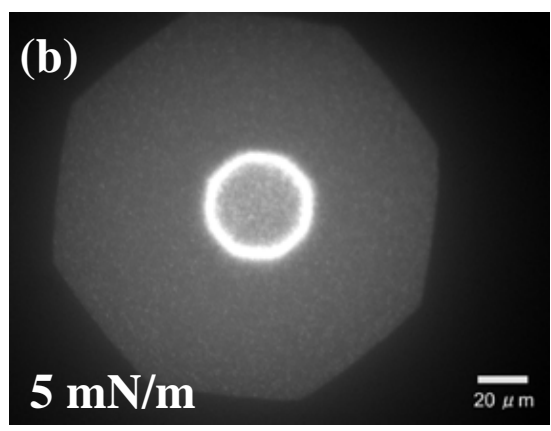

Fig. 2. O. Haruta, et al. 

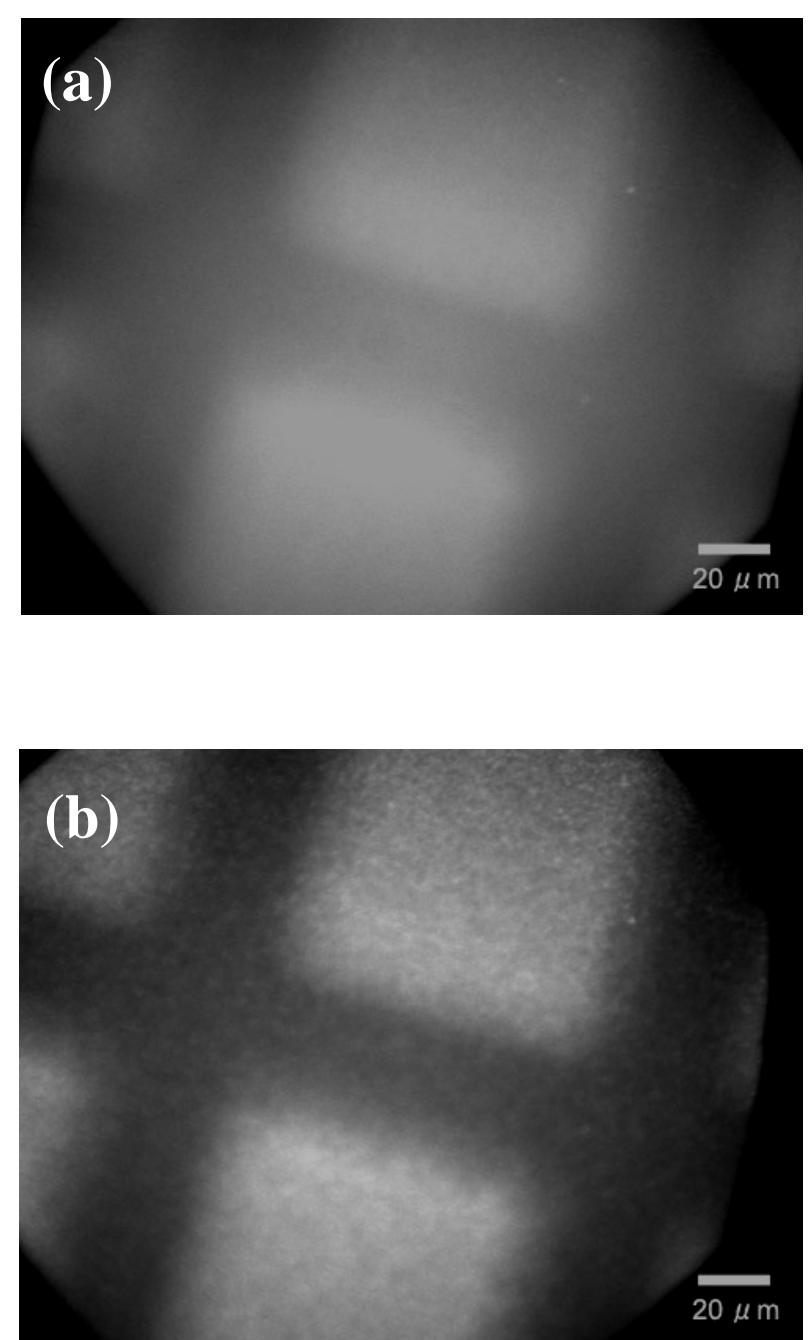

Fig. 3. O. Haruta, et al. 

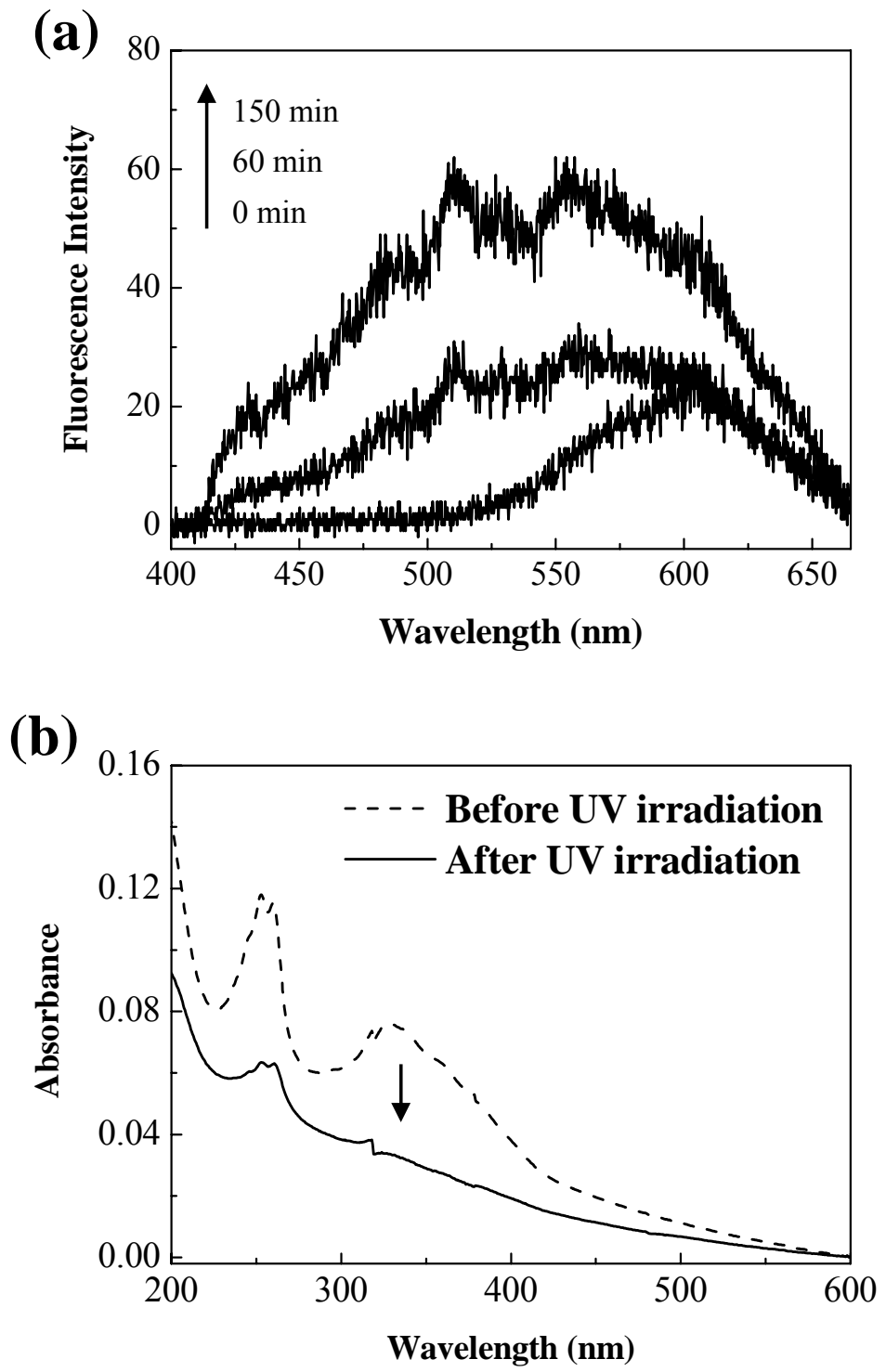

Fig. 4. O. Haruta, et al. 